\title{
Relative Orbit Stabilization Control for the Agile Satellite under Stochastic Disturbance
}

\author{
Xiande Wu, ${ }^{1}$ Fengzhi Guo, ${ }^{1}$ Wenbo Yang, ${ }^{2,3}$ Jiangtao $\mathrm{Xu},{ }^{1}$ and Ting Song ${ }^{2}$ \\ ${ }^{1}$ College of Aerospace and Civil Engineering, Harbin Engineering University, Harbin 150001, China \\ ${ }^{2}$ Shanghai Institute of Space Flight Control Technology, Shanghai 200030, China \\ ${ }^{3}$ Shanghai Key Laboratory of Aerospace Intelligent Control Technology, Shanghai 200030, China \\ Correspondence should be addressed to Xiande Wu; xiande_wu@163.com
}

Received 2 January 2014; Accepted 8 February 2014; Published 23 March 2014

Academic Editor: Ming Liu

Copyright (c) 2014 Xiande Wu et al. This is an open access article distributed under the Creative Commons Attribution License, which permits unrestricted use, distribution, and reproduction in any medium, provided the original work is properly cited.

This paper investigates the relative orbit control problem for a space communication satellite network. An observer-based state feedback control scheme is developed under the circumstance of faults and disturbance occurring in the sensors and actuators. The validity of sliding mode observer for the satellites' network is deduced and the analysis and proof of the relative orbit stabilization control are completed.

\section{Introduction}

The agile satellites have brought a great number of conveniences for modern spatial application; they have evolved from single satellite to constellation and formation [1]; furthermore, a spatial dynamic network is constructed. In order to expand the range of imaging services, orbit maneuver ability becomes an intrinsic ability of the satellite, and the satellites do not run on their preselected orbit. Therefore, the relative position and velocity need to be measured; all the members in the spatial agile imaging network need to be controlled real-timely [2].

The research of relative motion control is focusing on the two aspects, namely, relative orbit control and relative attitude control. Some methods are proposed for relative orbit control, such as optimal control for the orbit rendezvous [3] and predictive control for the rendezvous maneuver [4]. The relative control methods include the centralized and decentralized approach [5]. These methods pay more attention to the control law design in an ideal world where the status measurement sensors and control actuators work well. The modern control method is mentioned and analyzed [6-11] while, in the practical orbit control, the sensors and actuators will work in the fault or disturbance status.

This paper will complete the studies and analysis of sliding mode observer and state feedback control based on designed observer. The relative motion dynamic model will be depicted in Section 2; the sliding mode observer will be discussed in Section 3; the state feedback controller will be designed and its stabilization analysis is completed in Section 4.

\section{Dynamic Model of Network Members}

The relative motion dynamics of satellite communication network is usually established in the local-vertical-local horizontal [LVLH] coordinate system [12]. The relative motion equation can be rewritten as in the following form:

$$
\ddot{x}(t)-A_{1} \dot{x}-A_{2} x-C(x)=B u \text {. }
$$

Formula (1) can be expanded into the following form:

$$
\left[\begin{array}{c}
\ddot{x} \\
\ddot{y} \\
\ddot{z}
\end{array}\right]-\left[\begin{array}{ccc}
0 & 0 & 2 \omega_{t} \\
0 & 0 & 0 \\
-2 \omega_{t} & 0 & 0
\end{array}\right]\left[\begin{array}{c}
\dot{x} \\
\dot{y} \\
\dot{z}
\end{array}\right]-\left[\begin{array}{ccc}
\omega_{t}^{2} & 0 & \dot{\omega}_{t} \\
0 & 0 & 0 \\
-\dot{\omega}_{t} & 0 & \omega_{t}^{2}
\end{array}\right]\left[\begin{array}{l}
x \\
y \\
z
\end{array}\right]
$$




$$
-\left[\begin{array}{c}
-\frac{\mu}{r_{t}^{3}} \delta x \\
-\frac{\mu}{r_{t}^{3}} \delta y \\
-\frac{\mu}{r_{t}^{3}} \delta z+\frac{\mu}{r_{t}^{2}} \delta-\frac{\mu}{r_{t}^{2}}
\end{array}\right]=\left[\begin{array}{c}
a_{x} \\
a_{y} \\
a_{z}
\end{array}\right]
$$

The $x, y$, and $z$ are the relative coordinates to the target spacecraft, $\omega$ is the orbit angular of the target spacecraft, $r_{t}$ is the orbit radius of the spacecrafts, $a_{x}, a_{y}, a_{z}$ is the control acceleration, $\mu$ is the gravitational constant, and $\delta=\left[\left(x^{2} / r_{t}^{2}\right)+\left(y^{2} / r_{t}^{2}\right)+\left(z / r_{t}-1\right)^{2}\right]^{-3 / 2}$. Actually (2) is equivalent to

$$
\begin{gathered}
\ddot{x}(t)-2 \omega_{t} \dot{z}-\omega_{t}^{2} x-\dot{\omega}_{t} z+\frac{\mu}{r_{t}^{3}} \delta x=a_{x}, \\
\ddot{y}(t)+\frac{\mu}{r_{t}^{3}} \delta y=a_{y}, \\
\ddot{z}(t)+2 \omega_{t} \dot{x}+\dot{\omega}_{t} x-\omega_{t}^{2} z+\frac{\mu}{r_{t}^{3}} \delta z-\frac{\mu}{r_{t}^{2}} \delta+\frac{\mu}{r_{t}^{2}}=a_{z} .
\end{gathered}
$$

Hence $\ddot{x}(t), \ddot{y}(t)$, and $\ddot{z}(t)$ can be derived, and the expressions, respectively, are

$$
\begin{gathered}
\ddot{x}(t)=2 \omega_{t} \dot{z}+\omega_{t}^{2} x+\dot{\omega}_{t} z-\frac{\mu}{r_{t}^{3}} \delta x+a_{x}, \\
\ddot{y}(t)=-\frac{\mu}{r_{t}^{3}} \delta y+a_{y}, \\
\ddot{z}(t)=-2 \omega_{t} \dot{x}-\dot{\omega}_{t} x+\omega_{t}^{2} z-\frac{\mu}{r_{t}^{3}} \delta z+\frac{\mu}{r_{t}^{2}} \delta-\frac{\mu}{r_{t}^{2}}+a_{z} .
\end{gathered}
$$

Consider each expression of variables in the system (4):

$$
\begin{gathered}
x=\left[\begin{array}{l}
x \\
y \\
z
\end{array}\right], \quad u=\left[\begin{array}{l}
a_{x} \\
a_{y} \\
a_{z}
\end{array}\right], \quad A_{1}=\left[\begin{array}{ccc}
0 & 0 & 2 \omega_{t} \\
0 & 0 & 0 \\
-2 \omega_{t} & 0 & 0
\end{array}\right], \\
A_{2}=\left[\begin{array}{ccc}
\omega_{t}^{2} & 0 & \dot{\omega}_{t} \\
0 & 0 & 0 \\
-\dot{\omega}_{t} & 0 & \omega_{t}^{2}
\end{array}\right], \\
C(x)=\left[\begin{array}{c}
-\frac{\mu}{r_{t}^{3}} \delta x \\
-\frac{\mu}{r_{t}^{3}} \delta y \\
-\frac{\mu}{r_{t}^{3}} \delta z+\frac{\mu}{r_{t}^{2}} \delta+\frac{\mu}{r_{t}^{2}}
\end{array}\right], \quad B=\left[\begin{array}{lll}
1 & 0 & 0 \\
0 & 1 & 0 \\
0 & 0 & 1
\end{array}\right] .
\end{gathered}
$$

We define augmented variable as follows:

$$
x_{a}(t)=\left[\begin{array}{c}
x \\
y \\
z \\
\dot{x} \\
\dot{y} \\
\dot{z}
\end{array}\right] .
$$

The system (4) can be rewritten as in the following form:

$$
\begin{aligned}
& \dot{x}_{a}(t)=\left[\begin{array}{c}
\dot{x} \\
\dot{y} \\
\dot{z} \\
\ddot{x} \\
\ddot{y} \\
\ddot{z}
\end{array}\right]=\left[\begin{array}{cccccc}
0 & 0 & 0 & 1 & 0 & 0 \\
0 & 0 & 0 & 0 & 1 & 0 \\
0 & 0 & 0 & 0 & 0 & 1 \\
\omega_{t}^{2}-\frac{\mu}{r_{t}^{3}} \delta & 0 & \dot{\omega}_{t} & 0 & 0 & 2 \omega_{t} \\
0 & 0 & -\frac{\mu}{r_{t}^{3}} \delta & 0 & 0 & 0 \\
-\dot{\omega}_{t} & 0 & \omega_{t}^{2}-\frac{\mu}{r_{t}^{3}} \delta & -2 \omega_{t} & 0 & 0
\end{array}\right]\left[\begin{array}{c}
x \\
y \\
z \\
\dot{x} \\
\dot{y} \\
\dot{z}
\end{array}\right] \\
& +\left[\begin{array}{lll}
0 & 0 & 0 \\
0 & 0 & 0 \\
0 & 0 & 0 \\
1 & 0 & 0 \\
0 & 1 & 0 \\
0 & 0 & 1
\end{array}\right]\left[\begin{array}{c}
a_{x} \\
a_{y} \\
a_{z}
\end{array}\right]+\left[\begin{array}{c}
0 \\
0 \\
0 \\
0 \\
0 \\
\frac{\mu}{r_{t}^{2}} \delta-\frac{\mu}{r_{t}^{2}}
\end{array}\right] .
\end{aligned}
$$

Aiming at system (7), we consider more complex and practical situation: there exist sensors and actuators fault, constant input disturbance (namely, the last item in formula (7)), and the output disturbance in the system. Then we define system matrix as follows:

$$
\begin{gathered}
A=\left[\begin{array}{cccccc}
0 & 0 & 0 & 1 & 0 & 0 \\
0 & 0 & 0 & 0 & 1 & 0 \\
0 & 0 & 0 & 0 & 0 & 1 \\
\omega_{t}^{2}-\frac{\mu}{r_{t}^{3}} \delta & 0 & \dot{\omega}_{t} & 0 & 0 & 2 \omega_{t} \\
0 & 0 & -\frac{\mu}{r_{t}^{3}} \delta & 0 & 0 & 0 \\
-\dot{\omega}_{t} & 0 & \omega_{t}^{2}-\frac{\mu}{r_{t}^{3}} \delta & -2 \omega_{t} & 0 & 0
\end{array}\right], \\
\left.B=\left[\begin{array}{lll}
0 & 0 & 0 \\
0 & 0 & 0 \\
0 & 0 & 0 \\
1 & 0 & 0 \\
0 & 1 & 0 \\
0 & 0 & 1
\end{array}\right], \begin{array}{c}
u(t)=\left[\begin{array}{c}
a_{x} \\
a_{y} \\
a_{z}
\end{array}\right], \\
B_{\omega 1}
\end{array}\right]\left[\begin{array}{c}
0 \\
0 \\
0 \\
0 \\
0 \\
\frac{\mu}{r_{t}^{2}} \delta-\frac{\mu}{r_{t}^{2}}
\end{array}\right] .
\end{gathered}
$$

The system (7) can be rewritten as

$$
\begin{aligned}
\dot{x}(t)= & A x(t)+B u(t)+B_{\omega 1} \omega(t)+B_{a} f_{a}(t), \\
y(t)= & C x(t)+D u(t)+D_{a} f_{a}(t)+C_{s} f_{s}(t), \\
& +D_{d} d(t)+B_{\omega 2} \omega(t) .
\end{aligned}
$$

Here, $B_{a} \in R^{n \times a}, D_{a} \in R^{p \times a}, C_{s} \in R^{p \times s}, D_{d} \in R^{p \times d}$, and $B_{\omega 2} \in R^{p \times 1}$ represent system matrix and $f_{a}(t) \in R^{a}$ 
and $f_{s}(t) \in R^{s}$, respectively, are actuators fault and sensors fault. $d(t) \in R^{d}$ is the sensors' disturbance. $\omega(t)$ is the constant disturbance. Here we pay attention to a more general situation: the disturbance $\omega(t)$ meanwhile exists in the state equation and output $y(t)$ of system (9).

Defining $\bar{n}=n+a+\omega+2 p$, we do the following assumptions to the system (9).

(A1) fault and the perturbation vector: $f_{a}(t), f_{s}(t), d(t)$, and $\omega(t)$ satisfy the following assumption:

$$
\begin{array}{cl}
\left\|f_{s}(t)\right\| \leq r_{s 1}, & \left\|\dot{f}_{s}(t)\right\| \leq r_{s 2}, \\
\left\|f_{a}(t)\right\| \leq r_{a 1}, & \left\|\dot{f}_{a}(t)\right\| \leq r_{a 2}, \\
\|\omega(t)\| \leq r_{\omega 1}, & \|\dot{\omega}(t)\| \leq r_{\omega 2}, \\
\|d(t)\| \leq r_{d 1}, &
\end{array}
$$

where $r_{s 1}>0, r_{s 2}>0, r_{a 1}>0, r_{a 2}>0, r_{\omega 1}>0$, $r_{\omega 2}>0$, and $r_{d}>0$ are known constant.

(A2) $(A, C)$ is able to be observed, and there exists constant $a>0$, which makes

$$
\operatorname{rank}\left[\begin{array}{cc}
a I_{n}+A & B_{a} \\
C & D_{a}
\end{array}\right]=\bar{n} .
$$

(A3) matrix: $D_{a}, C_{s}, D_{d}, B_{\omega 2}$ are column full rank matrix.

For the convenience of discussion, we define augmented vector and matrix as follows:

$$
\bar{A}=\left[\begin{array}{ccccc}
A & 0 & 0 & 0 & 0 \\
0 & -\alpha I_{a} & 0 & 0 & 0 \\
0 & 0 & -\alpha I_{\omega} & 0 & 0 \\
0 & 0 & 0 & -\alpha I_{p} & 0 \\
0 & 0 & 0 & 0 & -I_{p}
\end{array}\right]
$$$$
\bar{B}=\left[\begin{array}{c}
B_{n \times m} \\
0_{a \times m} \\
0_{\omega \times m} \\
0_{p \times m} \\
0_{d \times m}
\end{array}\right] \bar{x}(t)=\left[\begin{array}{c}
x(t) \\
f_{a}(t) \\
\omega(t) \\
C_{s} f_{s}(t) \\
D_{d} d(t)
\end{array}\right] \text {, }
$$$$
\bar{B}_{a}=\left[\begin{array}{ccc}
\alpha^{-1} B_{a} & \alpha^{-1} B_{\omega} & 0_{n \times s} \\
I_{a} & 0_{a \times \omega} & 0 \\
0 & I_{\omega} & 0 \\
0 & 0 & C_{s} \\
0 & 0 & 0
\end{array}\right]
$$

$$
f(t)=\left[\begin{array}{c}
\left(\alpha f_{a}(t)+\dot{f}_{a}(t)\right)_{a \times 1} \\
(\alpha \omega(t)+\dot{\omega}(t))_{\omega \times 1} \\
\left(\alpha f_{s}(t)+\dot{f}_{s}(t)\right)_{s \times 1}
\end{array}\right], \quad \bar{N}=\left[\begin{array}{c}
0_{n} \\
0_{a} \\
0_{\omega} \\
0_{p} \\
I_{p}
\end{array}\right],
$$

$$
\bar{E}=\left[\begin{array}{ccccc}
I_{n} & \alpha^{-1} B_{a} & 0 & 0 & 0 \\
0 & I_{a} & 0 & 0 & 0 \\
0 & 0 & I_{\omega} & 0 & 0 \\
0 & 0 & 0 & I_{p} & 0 \\
0 & 0 & 0 & 0 & 0
\end{array}\right], \quad \bar{C}=\left[\begin{array}{lllll}
C & D_{a} & B_{\omega 2} & I_{p} & I_{p}
\end{array}\right]
$$

Establish an augmented generalized system based on system (9) as follows:

$$
\begin{gathered}
\bar{E} \dot{\bar{x}}(t)=\bar{A} x(t)+\bar{B} u(t)+\bar{B}_{a} f(t)+\bar{N} D_{d} d(t), \\
y(t)=\bar{C} \bar{x}(t)+D u(t) .
\end{gathered}
$$

Matrix $\bar{E}$ and matrix $\bar{C}$ have the following properties:

$$
\begin{aligned}
\operatorname{rank}[\bar{E} \bar{C}] & =\left[\begin{array}{ccccc}
I_{n} & \alpha^{-1} B_{a} & 0 & 0 & 0 \\
0 & I_{a} & 0 & 0 & 0 \\
0 & 0 & I_{\omega} & 0 & 0 \\
0 & 0 & 0 & I_{p} & 0 \\
0 & 0 & 0 & 0 & 0 \\
C & D_{a} & B_{\omega 2} & I_{p} & I_{p}
\end{array}\right] \\
& =n+a+\omega+p+p=\bar{n} .
\end{aligned}
$$

Therefore, it can be inferred that, according to matrix knowledge, there must be an appropriate number of dimensions matrix $\bar{L}$ which makes $\bar{E}+\overline{L C}$ an invertible matrix. We may define a new matrix

$$
\bar{L}_{D}=\left[\begin{array}{c}
0_{n \times p} \\
0_{a \times p} \\
0_{\omega \times p} \\
0_{p \times p} \\
\bar{L}_{D}^{(4)}
\end{array}\right]
$$

where $\bar{L}_{D}^{(4)} \in R^{p \times p}$ and $\bar{L}_{D}^{(4)}=\operatorname{diag}\left\{\begin{array}{llll}\beta_{1} & \beta_{2} & \cdots & \beta_{p}\end{array}\right\}, \beta_{i}>0$, $i>1,2, \ldots, p$. Meanwhile, we define a new matrix $\bar{S}=\bar{E}+$ $\bar{L}_{D} \bar{C}$. We can calculate directly

$$
\begin{aligned}
\bar{S} & =\left[\begin{array}{ccccc}
I_{n} & \alpha^{-1} B_{a} & 0 & 0 & 0 \\
0 & I_{a} & 0 & 0 & 0 \\
0 & 0 & I_{\omega} & 0 & 0 \\
0 & 0 & 0 & I_{p} & 0 \\
0 & 0 & 0 & 0 & 0
\end{array}\right]+\left[\begin{array}{c}
0_{n \times p} \\
0_{a \times p} \\
0_{\omega \times p} \\
0_{p \times p} \\
\bar{L}_{D}^{(4)}
\end{array}\right]\left[\begin{array}{lllll}
C & D_{a} & B_{\omega 2} & I_{p} & I_{p}
\end{array}\right] \\
& =\left[\begin{array}{ccccc}
I_{n} & \alpha^{-1} B_{a} & 0 & 0 & 0 \\
0 & I_{a} & 0 & 0 & 0 \\
0 & 0 & I_{\omega} & 0 & 0 \\
0 & 0 & 0 & I_{p} & 0 \\
0 & 0 & 0 & 0 & 0
\end{array}\right]
\end{aligned}
$$




$$
\begin{gathered}
+\left[\begin{array}{rcccc}
0 & 0 & 0 & 0 & 0 \\
0 & 0 & 0 & 0 & 0 \\
0 & 0 & 0 & 0 & 0 \\
0 & 0 & 0 & 0 & 0 \\
\bar{L}_{D}^{(4)} C & \bar{L}_{D}^{(4)} D_{a} & \bar{L}_{D}^{(4)} B_{\omega 2} & \bar{L}_{D}^{(4)} & \bar{L}_{D}^{(4)}
\end{array}\right] \\
=\left[\begin{array}{ccccc}
I_{n} & \alpha^{-1} B_{a} & 0 & 0 & 0 \\
0 & I_{a} & 0 & 0 & 0 \\
0 & 0 & I_{\omega} & 0 & 0 \\
0 & 0 & 0 & I_{P} & 0 \\
\bar{L}_{D}^{(4)} C & \bar{L}_{D}^{(4)} D_{a} & \bar{L}_{D}^{(4)} B_{\omega 2} & \bar{L}_{D}^{(4)} & \bar{L}_{D}^{(4)}
\end{array}\right] .
\end{gathered}
$$

We can conclude that there must be $\bar{S}^{-1}$ according to (16). Suppose that $\bar{S}^{-1}$ has the following form:

$$
\bar{S}^{-1}=\left[\begin{array}{ccccc}
I_{n} & -\alpha^{-1} B_{a} & 0 & 0 & 0 \\
0 & I_{a} & 0 & 0 & 0 \\
0 & 0 & I_{\omega} & 0 & 0 \\
0 & 0 & 0 & I_{p} & 0 \\
X_{1} & X_{2} & X_{3} & X_{4} & X_{5}
\end{array}\right] .
$$

In formula (17), $X_{1}, X_{2}, X_{3}, X_{4}$, and $X_{5}$ are matrixes needed to be solved. Expand $\overline{S S}^{-1}=I_{\bar{n}}$ as follows:

$$
\begin{gathered}
{\left[\begin{array}{ccccc}
I_{n} & \alpha^{-1} B_{a} & 0 & 0 & 0 \\
0 & I_{a} & 0 & 0 & 0 \\
0 & 0 & I_{\omega} & 0 & 0 \\
0 & 0 & 0 & I_{p} & 0 \\
\bar{L}_{D}^{(4)} C & \bar{L}_{D}^{(4)} D_{a} & \bar{L}_{D}^{(4)} B_{\omega 2} & \bar{L}_{D}^{(4)} & \bar{L}_{D}^{(4)}
\end{array}\right]} \\
\times \\
{\left[\begin{array}{ccccc}
I_{n} & -\alpha^{-1} B_{a} & 0 & 0 & 0 \\
0 & I_{a} & 0 & 0 & 0 \\
0 & 0 & I_{\omega} & 0 & 0 \\
0 & 0 & 0 & I_{p} & 0 \\
X_{1} & X_{2} & X_{3} & X_{4} & X_{5}
\end{array}\right]=I_{\bar{n}}}
\end{gathered}
$$

Consider the elements in the last line of the expression $\overline{S S}^{-1}=$ $I_{\bar{n}}$. Consider

$$
\begin{gathered}
\bar{L}_{D}^{(4)} C+\bar{L}_{D}^{(4)} X_{1}=0 \\
-\alpha^{-1} \bar{L}_{D}^{(4)} C B_{a}+\bar{L}_{D}^{(4)} D_{a}+\bar{L}_{D}^{(4)} X_{2}=0 \\
\bar{L}_{D}^{(4)} B_{\omega 2}+\bar{L}_{D}^{(4)} X_{3}=0 \\
\bar{L}_{D}^{(4)}+\bar{L}_{D}^{(4)} X_{4}=0 \\
\bar{L}_{D}^{(4)} X_{5}=I_{p} .
\end{gathered}
$$

Formula (2) can be obtained by directly calculating

$$
\begin{gathered}
X_{1}=-C, \\
X_{2}=\alpha^{-1} C B_{a}-D_{a}, \\
X_{3}=-B_{\omega 2},
\end{gathered}
$$

$$
\begin{gathered}
X_{4}=-I_{p}, \\
X_{5}=\left(\bar{L}_{D}^{(4)}\right)^{-1} .
\end{gathered}
$$

\section{Observer Design}

To get the asymptotic estimates of the state of system (9) and, at the same time, solve the corresponding control problem, we introduce sliding-mode observer as follows:

$$
\begin{aligned}
\bar{S} \dot{z}(t)= & \left(\bar{A}-\bar{L}_{p} \bar{C}\right) \bar{z}(t)-\bar{N}(y(t)-D u(t)) \\
& +\bar{B} u(t)+L_{s} u_{s}(t), \\
\widehat{\bar{x}}= & \bar{z}(t)+\bar{S}^{-1} \bar{L}_{D}(y(t)-D u(t)) .
\end{aligned}
$$

Here,

$$
z(t)=\left[\begin{array}{c}
z_{x}(t) \\
z_{a}(t) \\
z_{\omega}(t) \\
z_{s}(t) \\
z_{d}(t)
\end{array}\right], \quad \hat{\bar{x}}(t)=\left[\begin{array}{c}
\widehat{x}(t) \\
\widehat{f}_{a}(t) \\
\widehat{\omega}(t) \\
\widehat{f_{s}}(t) \\
\widehat{d}(t)
\end{array}\right]
$$

where $z_{x}(t) \in R^{n}, z_{a}(t) \in R^{a}, z_{\omega}(t) \in R^{\omega}, z_{s}(t) \in R^{p}$, $z_{d}(t) \in R^{p}, \widehat{x}(t) \in R^{n}, \widehat{f}_{a}(t) \in R^{a}, \widehat{\omega}(t) \in R^{\omega}, \widehat{f}_{s}(t) \in R^{p}$, $\widehat{d}(t) \in R^{p} \cdot \bar{L}_{D} \in R^{\bar{n} \times p}, \bar{L}_{s} \in R^{\bar{n} \times p}$, and $\bar{L}_{s} \in R^{\bar{n} \times p}$, respectively, are derivative gain, proportional gain, and sliding gain of the observer and $\bar{S}=\bar{E}+\bar{L}_{D} \bar{C}$ is defined previously. The $z_{s}(t)$ and $z_{d}(t)$ are not real estimation of $f_{s}(t)$ and $d(t)$ in the observer (21). Assume that the real estimations of $z_{s}(t)$ and $z_{d}(t)$, respectively, are $\breve{f}_{s}(t)$ and $\breve{d}(t)$; thus,

$$
\widehat{f}_{s}(t)=C_{s} \breve{f}_{s}(t), \quad \widehat{d}(t)=D_{d} \breve{d}(t) .
$$

According to the assumption (A3), $C_{s}$ and $D_{d}$ are column full rank, so $\left(\begin{array}{ll}C_{s} & C_{s}\end{array}\right)^{-1}$ and $\left(\begin{array}{lll}D_{d} & D_{d}\end{array}\right)^{-1}$ exist. It can be concluded from (23) that

$$
\begin{aligned}
& \breve{f}_{s}(t)=\left(\begin{array}{ll}
C_{s}^{T} & C_{s}
\end{array}\right)^{-1} C_{s}^{T} \widehat{f}_{s}(t), \\
& \breve{d}(t)=\left(\begin{array}{ll}
D_{d}^{T} & D_{d}
\end{array}\right)^{-1} D_{d}^{T} \widehat{d}(t) .
\end{aligned}
$$

Lemma 1. In the case of (A2), for the observer (21), there is a gain matrix $\bar{L}_{p}$, which makes $\bar{S}^{-1}\left(\bar{A}-\bar{L}_{p} \bar{C}\right)$ Hurwitz.

Proof. First, considering matrix $\bar{S}^{-1} \bar{A}$, for the finite dimensions matrix, there must exist a constant $\mu>0$, making $\operatorname{Re}\left[\lambda_{i}\left(\bar{S}^{-1} \bar{A}\right)\right]>-\mu,(i=1,2, \ldots, \bar{n})$, which means $\operatorname{Re}\left[\lambda_{i}\left(-\left(\mu I+\bar{S}^{-1} \bar{A}\right)\right)\right]<0,(i=1,2, \ldots, \bar{n})$. 
So, for arbitrary real number $\sigma \in R^{+}$, the following matrix rank relationship existed:

$$
\begin{aligned}
& \operatorname{rank}\left[\begin{array}{c}
\sigma I_{\bar{n}}-\bar{S}^{-1} \bar{A} \\
\bar{C}
\end{array}\right]=\operatorname{rank}\left[\begin{array}{cc}
\bar{S}^{-1} & 0 \\
0 & I_{p}
\end{array}\right]\left[\begin{array}{c}
\sigma\left(\bar{E}+\bar{L}_{D} \bar{C}\right)-\bar{A} \\
\bar{C}
\end{array}\right], \\
& \operatorname{rank}\left(\sigma\left(\bar{E}+\bar{L}_{D} \bar{C}\right)-\bar{A}\right) \\
& =\operatorname{rank}\left[\begin{array}{ccccc}
\sigma I_{n}-A & -\sigma \alpha^{-1} B_{a} & 0 & 0 & 0 \\
0 & \sigma I_{a}+\alpha I_{a} & 0 & 0 & 0 \\
0 & 0 & I_{\omega}+\alpha I_{\omega} & 0 & 0 \\
0 & 0 & 0 & I_{p}+\alpha I_{p} & 0 \\
\sigma \bar{L}_{D}^{(4)} C & \sigma \bar{L}_{D}^{(4)} D_{a} & \sigma \bar{L}_{D}^{(4)} B_{\omega 2} & \sigma \bar{L}_{D}^{(4)} & \sigma \bar{L}_{D}^{(4)}+I_{p}
\end{array}\right] .
\end{aligned}
$$

In formula (26), notice that, for any $\sigma \in R^{+}, \operatorname{rank}\left(\sigma \bar{L}_{D}^{(4)}+I_{p}\right)=$ $p$ always existed. Therefore,

$$
\begin{aligned}
\operatorname{rank} & \left(\sigma\left(\bar{E}+\bar{L}_{D} \bar{C}\right)-\bar{A}\right) \\
\quad & \operatorname{rank}\left[\begin{array}{cccc}
\sigma I_{n}-A & -\sigma \alpha^{-1} B_{a} & 0 & 0 \\
0 & \sigma I_{a}+\alpha I_{a} & 0 & 0 \\
0 & 0 & I_{\omega}+\alpha I_{\omega} & 0 \\
0 & 0 & 0 & I_{p}+\alpha I_{p}
\end{array}\right]+p .
\end{aligned}
$$

On the other hand, we notice that

$$
\begin{aligned}
& \operatorname{rank}(\sigma \bar{E}-\bar{A}) \\
& =\operatorname{rank}\left[\begin{array}{ccccc}
\sigma I-A & -\sigma \alpha^{-1} B_{a} & 0 & 0 & 0 \\
0 & -\sigma I_{a}+\alpha I_{a} & 0 & 0 & 0 \\
0 & 0 & \sigma I_{\omega}+\alpha I_{\omega} & 0 & 0 \\
0 & 0 & 0 & \sigma I_{p}+\alpha I_{p} & 0 \\
0 & 0 & 0 & 0 & I_{d}
\end{array}\right] \\
& =\operatorname{rank}\left[\begin{array}{cccc}
\sigma I-A & -\sigma \alpha^{-1} B_{a} & 0 & 0 \\
0 & -\sigma I_{a}+\alpha I_{a} & 0 & 0 \\
0 & 0 & \sigma I_{\omega}+\alpha I_{\omega} & 0 \\
0 & 0 & 0 & \sigma I_{p}+\alpha I_{p}
\end{array}\right]+d .
\end{aligned}
$$

So we can derive $\operatorname{rank}\left(\sigma\left(\bar{E}+\bar{L}_{D} \bar{C}\right)-\bar{A}\right)=\operatorname{rank}(\sigma \bar{E}-\bar{A})$. According to (26), we can derive the following formula:

$$
\begin{aligned}
& \operatorname{rank}\left[\begin{array}{c}
\sigma I_{\bar{n}}-\bar{S}^{-1} \bar{A} \\
\bar{C}
\end{array}\right] \\
& =\operatorname{rank}\left[\begin{array}{c}
\sigma\left(\bar{E}+\bar{L}_{D} \bar{C}\right)-\bar{A} \\
\bar{C}
\end{array}\right] \\
& =\operatorname{rank}\left[\begin{array}{c}
\sigma \bar{E}-\bar{A} \\
\bar{C}
\end{array}\right]
\end{aligned}
$$

$$
\begin{aligned}
& =\operatorname{rank}\left[\begin{array}{ccccc}
\sigma I_{n}-A & -\sigma \alpha^{-1} B_{a} & 0 & 0 & 0 \\
0 & -\sigma I_{a}+\alpha I_{a} & 0 & 0 & 0 \\
0 & 0 & \sigma I_{\omega}+\alpha I_{\omega} & 0 & 0 \\
0 & 0 & 0 & \sigma I_{p}+\alpha I_{p} & 0 \\
0 & 0 & 0 & 0 & I_{p} \\
C & D_{a} & B_{\omega 2} & I_{p} & I_{p}
\end{array}\right] \\
& =\operatorname{rank}\left[\begin{array}{ccccc}
\sigma I_{n}-A & -\sigma \alpha^{-1} B_{a} & 0 & 0 \\
0 & -\sigma I_{a}+\alpha I_{a} & 0 & 0 \\
0 & 0 & \sigma I_{\omega}+\alpha I_{\omega} & 0 \\
0 & 0 & 0 & \sigma I_{p}+\alpha I_{p} \\
C & D_{a} & B_{\omega 2} & I_{p}
\end{array}\right]+p .
\end{aligned}
$$

Then we discuss the values of $\sigma \neq-\alpha$ and $\sigma=-\alpha$, based on formula (29).

First, we consider the situation of $\sigma \neq-\alpha$ and, at this time, formula (29) can be equivalent to

$$
\operatorname{rank}\left[\begin{array}{cc}
\sigma I_{n}-A & -\sigma \alpha^{-1} B_{a} \\
C & D_{a}
\end{array}\right]+\omega+2 p .
$$
(A2)

We can draw the conclusion based on the assumption

$$
\operatorname{rank}\left[\begin{array}{c}
\sigma I_{\bar{n}}-\bar{S}^{-1} \bar{A} \\
\bar{C}
\end{array}\right]=n+a+\omega+2 p=\bar{n} .
$$

On the other hand, consider the condition of $\sigma=-\alpha$ and, at this moment, formula (28) turns into

$$
\operatorname{rank}\left[\begin{array}{cccc}
-\alpha I_{n}-A & B_{a} & 0 & 0 \\
C & D_{a} & B_{\omega 2} & I_{p}
\end{array}\right]+p .
$$

Notice that $B_{\omega 2}$ is column full rank and the above formula turns into

$$
\operatorname{rank}\left[\begin{array}{cc}
-\alpha I_{n}-A & B_{a} \\
C & D_{a}
\end{array}\right]+\omega+2 p=n+a+\omega+2 p=\bar{n} .
$$

Integrating the above two cases derived, we have proved that, for any $\sigma \in R^{+}, \operatorname{rank}\left(\sigma\left(\bar{E}+\bar{L}_{D} \bar{C}\right)-\bar{A}\right)=\bar{n}$. Hence, $\left[\bar{S}^{-1} \bar{A} \bar{C}\right]$ is a couple observed, and can elicit that $\left[\begin{array}{lll}-\bar{S}^{-1} & \bar{A} & \bar{C}\end{array}\right]$ can be observed. Therefore, there exists matrix $\bar{H}$, making $-\bar{S}^{-1} \bar{A}-\bar{H} \bar{C}$ Hurwitz (i.e., the eigenvalues of $-\bar{S}^{-1} \bar{A}-\bar{H} \bar{C}$ are all negative). Thus there must exist matrix $\bar{X}>0$ which makes

$$
-\left(\mu I_{\bar{n}}+\bar{S}^{-1} \bar{A}\right) \bar{X}-\bar{X}\left(\mu I_{\bar{n}}+\bar{S}^{-1} \bar{A}\right)=-\bar{C}^{T} \bar{C} .
$$

Let the proportion gain of observer (21) be equal to $\bar{L}_{p}=$ $\bar{S} \bar{X}^{-1} \bar{C}$; then, we can calculate

$$
\begin{gathered}
{\left[\mu I_{\bar{n}}+\bar{S}^{-1}\left(\bar{A}-\bar{L}_{p} \bar{C}\right)\right]^{T} \bar{X}+\bar{X}\left[\mu I_{\bar{n}}+\bar{S}^{-1}\left(\bar{A}-\bar{L}_{p} \bar{C}\right)\right]} \\
=-\bar{C}^{T} \bar{C} .
\end{gathered}
$$

Therefore, $\operatorname{Re}\left[\lambda_{i}\left(\bar{S}^{-1}\left(\bar{A}-\bar{L}_{p} \bar{C}\right)\right)\right]<-\mu,(i=1,2, \ldots, \bar{n})$. The proof completes. 


\section{Observer Error System}

Then we derive the error system of the observer (21). Firstly, in the first formula of the system (13), we add $\bar{L}_{D} \bar{C} \dot{\bar{x}}(t)$ at the left and right side and we can get

$$
\begin{aligned}
\left(\bar{E}+\bar{L}_{D} \bar{C}\right) \dot{\bar{x}}(t) & \\
=\bar{A} x(t)+ & \bar{B} u(t)+\bar{B}_{a} f(t)+\bar{L}_{D} \bar{C} \dot{\bar{x}}(t)+\bar{N} D_{d} d(t) \\
\Longleftrightarrow \bar{S} \dot{\bar{x}}(t)= & \bar{A} x(t)+\bar{B} u(t)+\bar{B}_{a} f(t) \\
& \quad+\bar{L}_{D} \bar{C} \dot{\bar{x}}(t)+\bar{N} D_{d} d(t) .
\end{aligned}
$$

On the other hand, for the observer (21), we can get $\bar{S} \dot{\bar{x}}(t)$

$$
\begin{aligned}
= & \bar{S} z(t)+\bar{L}_{D}(y(t)-D u(t)) \\
= & \left(\bar{A}-\bar{L}_{p} \bar{C}\right) \bar{z}(t)-\bar{N}(y(t)-D u(t))+\bar{B} u(t)+\bar{L}_{s} u_{s}(t) \\
& +\bar{L}_{D}(\dot{y}(t)-D \dot{u}(t)) \\
= & \left(\bar{A}-\bar{L}_{p} \bar{C}\right) \overline{\bar{x}}(t)-\left(\bar{A}-\bar{L}_{p} \bar{C}\right) \bar{S}^{-1} \bar{L}_{D}(y(t)-D u(t)) \\
& -\bar{N}(y(t)-D u(t))+\bar{B} u(t)+\bar{L}_{s} u_{s}(t) \\
& +\bar{L}_{D}(\dot{y}(t)-D \dot{u}(t)) \\
= & \left(\bar{A}-\bar{L}_{p} \bar{C}\right) \overline{\bar{x}}(t)-\bar{A} \bar{S}^{-1} \bar{L}_{D}(y(t)-D u(t)) \\
& +\bar{L}_{p} \bar{C} \bar{S}^{-1} \bar{L}_{D}(y(t)-D u(t))-\bar{N}(y(t)-D u(t))+\bar{B} u(t) \\
& +\bar{L}_{s} u_{s}(t)+\bar{L}_{D}(\dot{y}(t)-D \dot{u}(t)) \\
= & {\left[\left(\bar{A}-\bar{L}_{p} \bar{C}\right) \overline{\bar{x}}(t)+\bar{L}_{p i}(y(t)-D u(t))+\bar{B} u(t)+\bar{L}_{s} u_{s}(t)\right] } \\
& +\bar{L}_{D}(\dot{y}(t)-D \dot{u}(t)) .
\end{aligned}
$$

Notice that $y(t)-D u(t)=\bar{C} \bar{x}(t)$, so $\dot{y}(t)-D \dot{u}(t)=\bar{C} \dot{\bar{x}}(t)$. Therefore, from formula (36), we can obtain

$$
\begin{aligned}
\bar{S} \dot{\bar{x}}= & \left(\bar{A}-\bar{L}_{p} \bar{C}\right) \hat{\bar{x}}(t)+\bar{L}_{p} \bar{C} \bar{x}(t)+\bar{B} u(t) \\
& +\bar{L}_{s} u_{s}(t)+\bar{L}_{D} \bar{C} \dot{\bar{x}}(t) .
\end{aligned}
$$

We define error variable as follows:

$$
\bar{e}(t)=\hat{\bar{x}}(t)-\bar{x}(t)=\left[\begin{array}{c}
e_{x}(t) \\
e_{a}(t) \\
e_{\omega}(t) \\
e_{s}(t) \\
e_{d}(t)
\end{array}\right] .
$$

Meanwhile considering (36) and (38), we obtain

$$
\bar{S} \dot{\bar{e}}(t)=\left(\bar{A}-\bar{L}_{p} \bar{C}\right) \bar{e}(t)+\bar{L}_{s} u_{s}(t)-\bar{B}_{a} f(t)-\bar{N} D_{d} d(t) .
$$

This is equal to

$$
\begin{aligned}
\dot{\bar{e}}(t)= & \bar{S}^{-1}\left(\bar{A}-\bar{L}_{p} \bar{C}\right) \bar{e}(t)+\bar{S}^{-1} \bar{L}_{s} u_{s}(t)-\bar{S}^{-1} \bar{B}_{a} f(t) \\
& -\bar{S}^{-1} \bar{N} D_{d} d(t) .
\end{aligned}
$$

In formula (41),

$$
\bar{S}^{-1} \bar{N}=\left[\begin{array}{ccccc}
I_{n} & -\alpha^{-1} B_{a} & 0 & 0 & 0 \\
0 & I_{a} & 0 & 0 & 0 \\
0 & 0 & I_{\omega} & 0 & 0 \\
0 & 0 & 0 & I_{p} & 0 \\
X_{1} & X_{2} & X_{3} & X_{4} & X_{5}
\end{array}\right]\left[\begin{array}{c}
0_{n} \\
0_{a} \\
0_{\omega} \\
0_{p} \\
I_{p}
\end{array}\right]=\left[\begin{array}{c}
0 \\
0 \\
0 \\
0 \\
\left(\bar{L}_{D}^{(4)}\right)^{-1}
\end{array}\right] .
$$

Here,

$$
\bar{L}_{D}^{(4)}=\left(\begin{array}{ccc}
\beta_{1} & \cdots & 0 \\
\vdots & \ddots & \vdots \\
0 & \cdots & \beta_{p}
\end{array}\right) .
$$

Hence, if the value of $\beta_{i}(i=1,2, \ldots, p)$ is large enough, in the system (41), $\bar{S}^{-1} \bar{N}$ will become infinitesimal. Until now, we remove the influence of disturbance $D_{d} d(t)$ for the system stability.

On the other hand, for the $\bar{S}^{-1} \bar{L}_{s} u_{s}(t)-\bar{S}^{-1} \bar{B}_{a} f(t)$ of the system (41), we design $u_{s}(t)$ as the following form $(\rho>0$ is design parameter):

$$
\begin{gathered}
u_{s}(t)=-\left(\alpha r_{a 1}+r_{a 2}+\alpha r_{\omega 1}+r_{\omega 2}+\alpha r_{s 1}+r_{s 2}+p\right) \operatorname{sgn}(s(t)), \\
s(t)=\bar{B}_{a}^{T} \bar{S}^{-1} \bar{P} \bar{e}(t) \in R^{a+\omega+s},
\end{gathered}
$$

of which, $\bar{P}$ is Lyapunov matrix required and $\bar{P}>0$ satisfies

$$
\bar{B}_{a}^{T} \bar{S}^{-1} \bar{P}=\bar{M} \bar{C} .
$$

Here, $\bar{M} \in R^{(a+\omega+s) \times p}$ is matrix parameters waiting for being solved. Based on the above analysis, error system (41) changes as

$$
\dot{\bar{e}}(t)=\bar{S}^{-1}\left(\bar{A}-\bar{L}_{p} \bar{C}\right) \bar{e}(t)+\bar{S}^{-1} \bar{L}_{s} u_{s}(t)-\bar{S}^{-1} \bar{B}_{a} f(t) .
$$

The following section discusses the stability of error system (46) and then discusses stabilization problem of closed-loop system.

\section{The Stability Analysis of the Error System}

Theorem 2. For error system (46), let the sliding mode observer gain $\bar{L}_{s}=\bar{B}_{a}$, if there exists matrix $\bar{P}>0$ making the following matrix inequality established:

$$
\bar{P} \bar{S}^{-1}\left(\bar{A}-\bar{L}_{p} \bar{C}\right)+\left(\bar{A}-\bar{L}_{p} \bar{C}\right)^{T}\left(\bar{S}^{-1}\right)^{T} \bar{P}<0 .
$$

Then the system (46) states trajectory asymptotically stable convergence to the origin. 
Proof. For the system (46), defining Lyapunov function $v(t)=$ $\bar{e}^{T}(t) \bar{P} \bar{e}(t), \bar{P}>0$, along the system (46) state trajectory, we can calculate

$\dot{v}(t)$

$$
\begin{aligned}
& =2 \bar{e}^{T}(t) \bar{P} \dot{\bar{e}}(t) \\
& =2 \bar{e}^{T}(t) \bar{P}\left[\bar{S}^{-1}\left(\bar{A}-\bar{L}_{p} \bar{C}\right) \bar{e}(t)+\bar{S}^{-1} \bar{L}_{s} u_{s}(t)-\bar{S}^{-1} \bar{B}_{a} f(t)\right] \\
& \leq 2 \bar{e}^{T}(t) \bar{P} \bar{S}^{-1}\left(\bar{A}-\bar{L}_{p} \bar{C}\right) \bar{e}(t)+2 \bar{P} \bar{S}^{-1}\left(\bar{L}_{s} u_{s}(t)-\bar{B}_{a} f(t)\right) .
\end{aligned}
$$

Consider parts of above formula,

$$
\begin{aligned}
& 2 \bar{e}(t) \bar{P} \bar{S}^{-1}\left(\bar{L}_{s} u_{s}(t)-\bar{B}_{a} f(t)\right) \\
& \leq 2 \bar{e}^{T}(t) \bar{P} \bar{S}^{-1} \bar{L}_{s} u_{s}(t)-2 \bar{e}^{T}(t) \bar{P} \bar{S}^{-1} \bar{B}_{a} f(t) \\
&=2 \bar{e}^{T}(t) \bar{P}^{-1} \bar{B}_{a}\left(u_{s}(t)-f(t)\right) \\
& \leq-2 \bar{e}^{T}(t) \bar{P} \bar{S}^{-1} \bar{B}_{a}\left(\alpha r_{a 1}+r_{a 2}+\alpha r_{\omega 1}+r_{\omega 2}+\alpha r_{s 1}+r_{s 2}+p\right) \\
& \quad \times \operatorname{sgn}(s(t))+2\left\|\bar{e}^{T}(t) \bar{P} \bar{S}^{-1} \bar{B}_{a} f(t)\right\| \\
& \leq-2 s^{T}(t)\left(\alpha r_{a 1}+r_{a 2}+\alpha r_{\omega 1}+r_{\omega 2}+\alpha r_{s 1}+r_{s 2}+p\right) \\
& \quad \times \operatorname{sgn}(s(t))+2\left\|s^{T}(t)\right\|\|f(t)\| \\
&=-2|s(t)|\left(\alpha r_{a 1}+r_{a 2}+\alpha r_{\omega 1}+r_{\omega 2}+\alpha r_{s 1}+r_{s 2}+p\right) \\
& \times \operatorname{sgn}(s(t))+2\left\|s^{T}(t)\right\|\|f(t)\| .
\end{aligned}
$$

The following formation can be derived based on assumption (A1):

$$
\|f(t)\| \leq \alpha r_{a 1}+r_{a 2}+\alpha r_{\omega 1}+r_{\omega 2}+\alpha r_{s 1}+r_{s 2}+p .
$$

Therefore

$$
2 \bar{e}^{T}(t) \bar{P} \bar{S}^{-1}\left(\bar{L}_{s} u_{s}(t)-\bar{B}_{a} f(t)\right) \leq 0 .
$$

So we can derive

$$
\begin{aligned}
\dot{v}(t) \leq & 2 \bar{e}^{T}(t) \bar{P} \bar{S}^{-1}\left(\bar{A}-\bar{L}_{p} \bar{C}\right) \bar{e}(t) \\
\leq & \bar{e}^{T}(t)\left[\bar{P} \bar{S}^{-1}\left(\bar{A}-\bar{L}_{p} \bar{C}\right)\right. \\
& \left.+\left(\bar{A}-\bar{L}_{p} \bar{C}\right)^{T}\left(\bar{S}^{-1}\right)^{T} \bar{P}\right] \bar{e}(t)
\end{aligned}
$$

$$
\leq 0 \text {. }
$$

If $\bar{e}(t) \neq 0$. the inequality always holds. So the error system (46) is asymptotically stable. The proof completes.

\section{The Stabilization of Closed-Loop System}

Now we consider the stabilization problem of the closed-loop system based on the observer. Considering the system (9), we design a state feedback controller based on observer as follows:

$$
u(t)=k x(t)-B^{-1} B_{a} \widehat{f}_{a}(t)-B^{-1} B_{\omega 1} \widehat{\omega}(t) .
$$

Substitute formula (53) into the system (9) and we can obtain

$$
\begin{aligned}
\dot{x}(t)= & A x(t)+B\left(k x(t)-B^{-1} B_{a} \widehat{f}_{a}(t)-B^{-1} B_{\omega 1} \widehat{\omega}(t)\right) \\
& +B_{\omega 1} \omega(t)+B_{a} f_{a}(t) \\
= & A x(t)+B k x(t)-B_{a} \widehat{f}_{a}(t)-B_{\omega 1} \widehat{\omega}(t)+B_{\omega 1} \omega(t) \\
& +B_{a} f_{a}(t) \\
= & A x(t)+B k x(t)-B_{a} e_{a}(t)-B_{\omega 1} e_{\omega}(t) .
\end{aligned}
$$

Here, $e_{a}(t)=\widehat{f}_{a}(t)-f_{a}(t), e_{\omega}(t)=\widehat{\omega}(t)-\omega(t)$.

Formula (54) can be rewritten as

$$
\dot{x}(t)=(A+B k) x(t)-\bar{F} \bar{e}(t) .
$$

Here, $\bar{F}=\left[\begin{array}{lllll}0_{n \times n} & B_{a} & B_{\omega 1} & 0_{n \times p} & 0_{n \times p}\end{array}\right]$.

For the close-loop system (55) and the error system (46), they can construct the following system:

$$
\begin{gathered}
\dot{x}(t)=(A+B k) x(t)-\bar{F} \bar{e}(t), \\
\dot{\bar{e}}(t)=\bar{S}^{-1}\left(\bar{A}-\bar{L}_{p} \bar{C}\right) \bar{e}(t)+\bar{S}^{-1} \bar{L}_{s} u_{s}(t)-\bar{S}^{-1} \bar{B}_{a} f(t) .
\end{gathered}
$$

We present the following theorem.

Theorem 3. If there is symmetric positive definite matrix $Z \in$ $R^{\bar{n}}$ and the matrix $K \in R^{m \times \bar{n}}$, which makes the following constraint matrix established:

$$
(A+B K)^{T} Z+Z(A+B K)<0,
$$

then the system (56) is asymptotically stable.

Proof. For the system (56), we define Lyapunov function

$$
v_{x}(t)=x^{T}(t) Z x(t) .
$$

Here, $Z>0$ is a positive definite symmetric matrix waiting for being solved. Along the system (56) trajectory, we can directly calculate

$$
\begin{aligned}
\dot{v}_{x}(t)= & 2 x^{T}(t) Z \dot{x}(t) \\
= & 2 x^{T}(t) Z[(A+B k) x(t)-\bar{F} \bar{e}(t)] \\
= & x^{T}(t) Z\left[(A+B k)^{T} Z+Z(A+B k)\right] \\
& -2 x^{T}(t) Z \bar{F} \bar{e}(t) .
\end{aligned}
$$

Let $\Phi=(A+B K)^{T} Z+Z(A+B K)$ and then

$$
\begin{aligned}
\dot{v}_{x}(t) & \leq x^{T}(t) \Phi x(t)-2 x^{T}(t) Z \bar{F} \bar{e}(t) \\
& \leq \lambda_{\min }(\Phi)\|x(t)\|^{2}+2\|x(t)\|\|Z \bar{F} \bar{e}(t)\| .
\end{aligned}
$$


Here, $\lambda_{\min }(\Phi)$ represents the minimum eigenvalue of the matrix $\Phi$. We define a new Lyapunov function

$$
v_{0}(t)=v_{x}(t)+\theta v(t)
$$

Here, $\theta>0$ is a parameter waiting for design, $v(t)$ is defined in Theorem 2.

According to the proof in Theorem 2, we can obtain

$$
\dot{v}(t) \leq \varepsilon_{1}\|\bar{e}(t)\|^{2} .
$$

Here,

$\varepsilon_{1}=\lambda_{\min }\left(\bar{P} \bar{S}^{-1}\left(\bar{A}-\bar{L}_{p} \bar{C}\right)+\left(\bar{A}-\bar{L}_{p} \bar{C}\right)^{T}\left(\bar{S}^{-1}\right)^{T} \bar{P}\right)<0$.

In addition, the new parameters are defined as follows:

$$
\varepsilon_{2}=\lambda_{\min }(\Phi)<0, \quad \varepsilon_{3}=2\|Z \bar{F}\|
$$

Selecting parameters $\theta>0$ and let it satisfy

$$
\theta>\frac{\varepsilon_{3}^{2}}{\varepsilon_{1} \varepsilon_{2}} .
$$

Calculate formula (61) further and we can obtain

$$
\begin{aligned}
\dot{v}_{0}(t) \leq & \varepsilon_{2}\|x(t)\|^{2}+\varepsilon_{3}\|x(t)\|\|\bar{e}(t)\|+\theta \varepsilon_{1}\|\bar{e}(t)\|^{2} \\
\leq & \varepsilon_{2}\|x(t)\|^{2}+\varepsilon_{3}\|x(t)\|\|\bar{e}(t)\|+\frac{\varepsilon_{3}^{2}}{\varepsilon_{1} \varepsilon_{2}} \varepsilon_{1}\|\bar{e}(t)\|^{2} \\
\leq & \varepsilon_{2}\|x(t)\|^{2}+\varepsilon_{3}\|x(t)\|\|\bar{e}(t)\|+\frac{\varepsilon_{3}^{2}}{\varepsilon_{2}}\|\bar{e}(t)\|^{2} \\
\leq & 0.5 \varepsilon_{2}\|x(t)\|^{2}+\varepsilon_{3}\|x(t)\|\|\bar{e}(t)\|+0.5 \frac{\varepsilon_{3}^{2}}{\varepsilon_{2}}\|\bar{e}(t)\|^{2} \\
& +0.5 \frac{\varepsilon_{3}^{2}}{\varepsilon_{2}}\|\bar{e}(t)\|^{2}+0.5 \varepsilon_{2}\|x(t)\|^{2} \\
\leq & \left(\sqrt{\frac{\varepsilon_{2}}{2}}\|x(t)\|+\sqrt{\frac{\varepsilon_{3}^{2}}{2 \varepsilon_{2}}}\|\bar{e}(t)\|\right)^{2}+0.5 \frac{\varepsilon_{3}^{2}}{\varepsilon_{2}}\|\bar{e}(t)\|^{2} \\
& +0.5 \varepsilon_{2}\|x(t)\|^{2} \\
\leq & 0.5 \frac{\varepsilon_{3}^{2}}{\varepsilon_{2}}\|\bar{e}(t)\|^{2}+0.5 \varepsilon_{2}\|x(t)\|^{2} .
\end{aligned}
$$

Notice that $\varepsilon_{3}>0, \varepsilon_{2}<0$, so we have

$$
\dot{v}_{0}(t) \leq 0.5 \frac{\varepsilon_{3}^{2}}{\varepsilon_{2}}\|\bar{e}(t)\|^{2}+0.5 \varepsilon_{2}\|x(t)\|^{2}<0 .
$$
stable.

\section{Conclusions and Future Works}

The sliding mode observer and the state feedback controller is proposed and the controller's stabilization under stochastic disturbance is proved. The research provides a theoretical analysis of the controller design method based on sliding mode observer. In the future, we will give the simulation verification combined with the specific space mission.

\section{Conflict of Interests}

The authors declare that there is no conflict of interests regarding the publication of this paper.

\section{Acknowledgments}

This work is partially funded by the Fundamental Research Funds for the Central Universities (no. HEUCF021318), the Natural Science Foundation of Heilongjiang Province (no. A201312), the National Natural Science Funds (no. 11372080), the Harbin Science and Technology Innovation Talent Youth Fund (no. RC2013QN001007), and the Key Laboratory of Database and Parallel Computing, Heilongjiang Province.

\section{References}

[1] M. Der-Ming, H. Zuu-Chang, L. Tzung-Hang, and C. Bo-Jyun, "Design of a micro-satellite constellation for communication," Acta Astronautica, vol. 82, no. 1, pp. 54-59, 2013.

[2] Z. Guoqiang, H. Min, and Y. Hong, "Relative orbit estimation and formation keeping control of satellite formations in low Earth orbits," Acta Astronautica, vol. 76, no. 7-8, pp. 164-175, 2012.

[3] H. Peng, C. Yang, Y. Li, S. Zhang, and B. Chen, "Surrogatebased parameter optimization and optimal control for optimal trajectory of Halo orbit rendezvous," Aerospace Science and Technology, vol. 26, no. 1, pp. 176-184, 2012.

[4] E. N. Hartley, P. A. Trodden, A. G. Richards, and J. M. Maciejowski, "Model predictive control system design and implementation for spacecraft rendezvous," Control Engineering Practice, vol. 20, no. 7, pp. 695-713, 2012.

[5] I. Chang, S.-Y. Park, and K.-H. Choi, "Decentralized coordinated attitude control for satellite formation flying via the statedependent Riccati equation technique," International Journal of Non-Linear Mechanics, vol. 44, no. 8, pp. 891-904, 2009.

[6] S. Yin, H. Luo, and S. Ding, "Real-time implementation of faulttolerant control systems with performance optimization," IEEE Transactions on Industrial Electronics, vol. 64, no. 5, pp. 24022411, 2014.

[7] S. Yin, G. Wang, and H. Karimi, "Data-driven design of robust fault detection system for wind turbines," Mechatronics. In press.

[8] S. Yin, S. Ding, A. Haghani, and H. Hao, "Data-driven monitoring for stochastic systems and its application on batch process," International Journal of Systems Science, vol. 44, no. 7, pp. 13661376, 2013.

[9] S. Yin, S. Ding, A. Haghani, H. Hao, and P. Zhang, "A comparison study of basic datadriven fault diagnosis and process monitoring methods on the benchmark Tennessee Eastman process," Journal of Process Control, vol. 22, no. 9, pp. 1567-1581, 2012. 
[10] W. Huanqing, C. Bing, and L. Chong, "Adaptive neural tracking control for a class of stochastic nonlinear systems with unknown dead-zone," International Journal of Innovative Computing, Information and Control, vol. 9, no. 8, pp. 3257-3269, 2013.

[11] A. Leslie, M. Patricia, and C. Oscar, "Chemical optimization paradigm applied to a Fuzzy tracking controller for an autonomous mobile robot," International Journal of Innovative Computing, Information and Control, vol. 9, no. 5, pp. 20072018, 2013.

[12] W. Shunan, W. Zhigang, R. Gianmarco, and W. Rui, "Adaptive control for spacecraft relative translation with parameteric uncertainty," Aerospace Science and Technology, vol. 31, no. 1, pp. 53-58, 2013. 


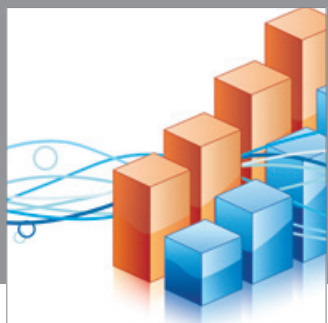

Advances in

Operations Research

mansans

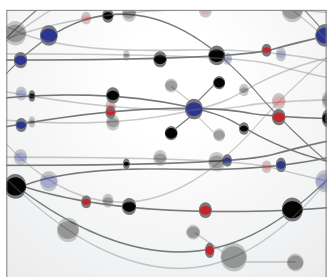

The Scientific World Journal
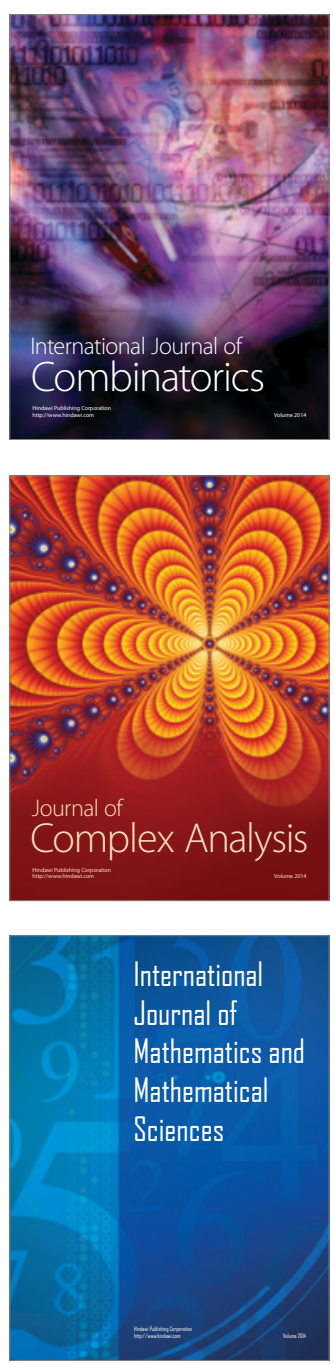
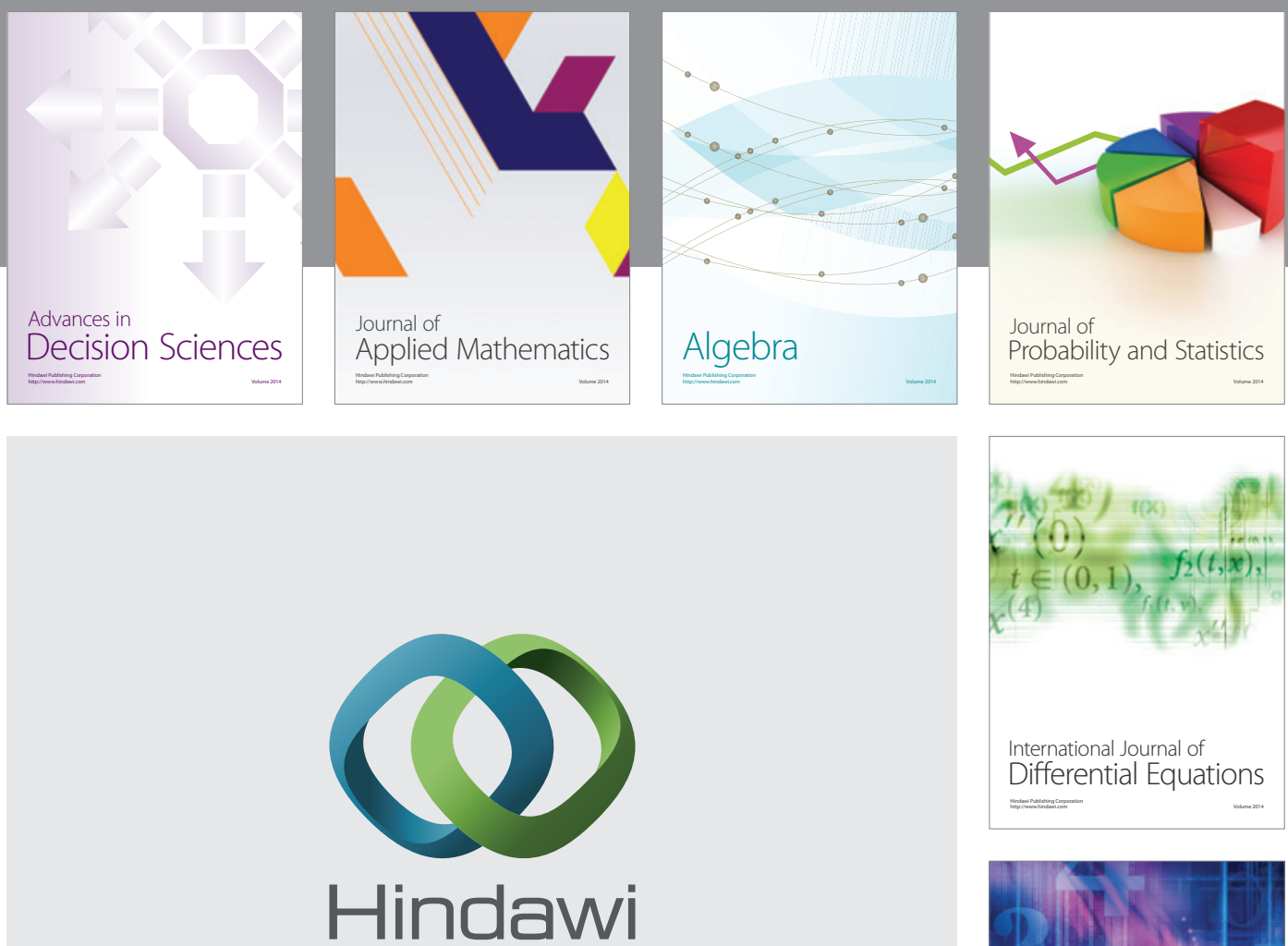

Submit your manuscripts at http://www.hindawi.com
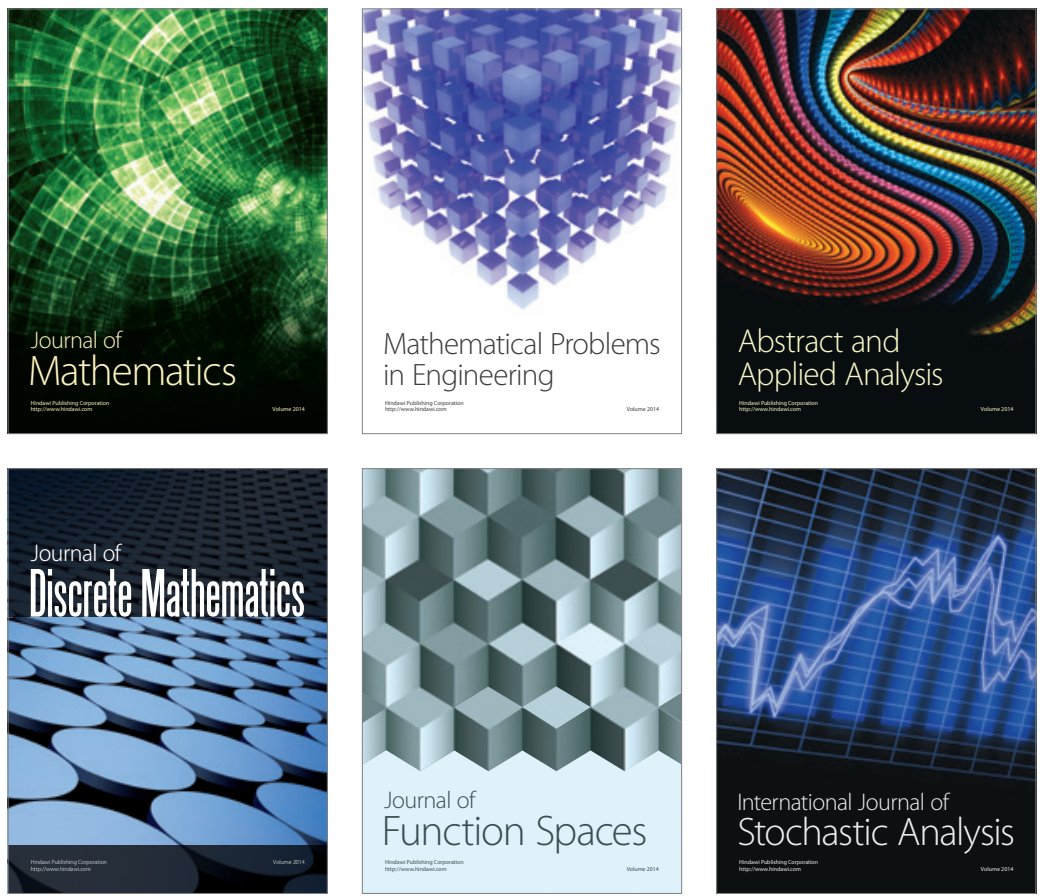

Journal of

Function Spaces

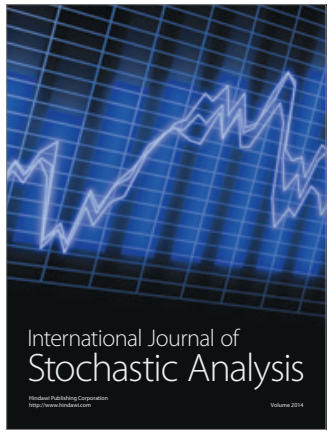

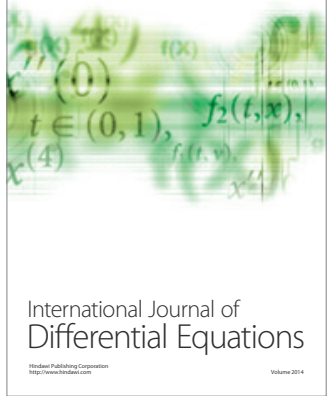
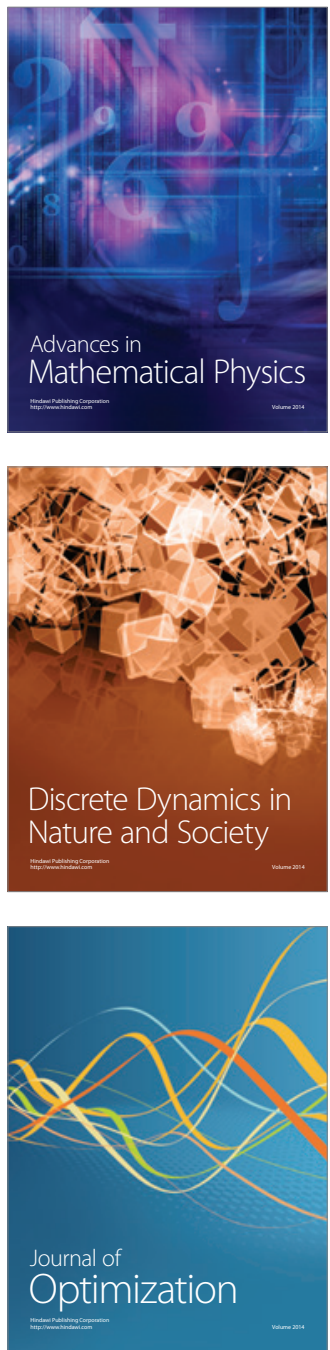